\title{
DYNAMICAL PROPERTIES OF THE WEIL-PETERSSON METRIC
}

\author{
URSULA HAMENSTÄDT
}

\begin{abstract}
Let $\mathcal{T}(S)$ be the Teichmüller space of an oriented surface $S$ of finite type. We discuss the action of subgroups of the mapping class group of $S$ on the CAT(0)-boundary of the completion of $\mathcal{T}(S)$ with respect to the Weil-Petersson metric. We show that the set of invariant Borel probability measures for the Weil-Petersson flow on moduli space which are supported on a closed orbit is dense in the space of all ergodic invariant probability measures.
\end{abstract}

\section{INTRODUCTION}

For an oriented surface $S$ of genus $g \geq 0$ with $m \geq 0$ punctures and complexity $3 g-3+m \geq 2$ let $\mathcal{T}(S)$ be the Teichmüller space of all isotopy classes of complete hyperbolic metrics on $S$ of finite volume. Then $\mathcal{T}(S)$ is a contractible manifold which can be equipped with the Weil-Petersson metric, an incomplete Kähler metric of negative sectional curvature.

In spite of the lack of completeness, any two points in $\mathcal{T}(S)$ can be connected by a unique Weil-Petersson geodesic which depends smoothly on its endpoints [8]. As a consequence, $\mathcal{T}(S)$ can be completed to a Hadamard space $\overline{\mathcal{T}(S)}$, i.e. a complete simply connected CAT(0)-space which however is not locally compact.

A Hadamard space $X$ admits a visual boundary $\partial X$, and the action of the isometry group of $X$ extends to an action on $\partial X$. For surfaces $S$ of complexity at most three, the visual boundary $\partial \overline{\mathcal{T}(S)}$ of $\overline{\mathcal{T}(S)}$ was identified by Brock and Masur [6], but for higher complexity it is not known. However, it follows from the work of Brock [5] that the boundary is not locally compact.

The extended mapping class group $\operatorname{Mod}(S)$ of all isotopy classes of diffeomorphisms of $S$ acts on $\mathcal{T}(S)$ as a group of isometries. Since every isometry of $\mathcal{T}(S)$ extends to an isometry of the completion $\overline{\mathcal{T}(S)}$, the extended mapping class group also acts isometrically on $\overline{\mathcal{T}(S)}$. This fact was used by Masur and Wolf 13 to show that in fact every isometry of $\overline{\mathcal{T}(S)}$ is an extended mapping class.

An isometry $g$ of $\overline{\mathcal{T}(S)}$ is called axial if $g$ admits an axis, i.e. if there is a geodesic $\gamma: \mathbb{R} \rightarrow \overline{\mathcal{T}(S)}$ and a number $\tau>0$ such that $g \gamma(t)=\gamma(t+\tau)$ for all $t$. The endpoints $\gamma(\infty), \gamma(-\infty)$ are then fixed points for the action of $g$ on $\partial \overline{\mathcal{T}(S)}$. Every pseudo-Anosov mapping class $g \in \operatorname{Mod}(S)$ is axial [8]. The limit set $\Lambda$ of a subgroup $G$ of $\operatorname{Mod}(S)$ is the set of accumulation points in $\partial \overline{\mathcal{T}(S)}$ of an orbit of the action of $G$ on $\overline{\mathcal{T}(S)}$. The group $G$ is called non-elementary if its limit set contains at least three points. We show.

Date: January 26, 2008.

Partially supported by Sonderforschungsbereich 611 . 
Theorem 1. Let $G<\operatorname{Mod}(S)$ be a non-elementary subgroup with limit set $\Lambda$ which contains a pseudo-Anosov element.

(1) $\Lambda$ does not have isolated points, and the $G$-action on $\Lambda$ is minimal.

(2) Pairs of fixed points of pseudo-Anosov elements are dense in $\Lambda \times \Lambda$.

(3) There is a dense orbit for the action of $G$ on $\Lambda \times \Lambda$.

There is a compactification of $\mathcal{T}(S)$ whose boundary consists of the sphere $\mathcal{P} \mathcal{M L}$ of projective measured geodesic laminations on $S$. The mapping class group acts on $\mathcal{T}(S) \cup \mathcal{P} \mathcal{M L}$ as a group of homeomorphisms. Limit sets for subgroups of $\operatorname{Mod}(S)$ in $\mathcal{P} \mathcal{M L}$ were investigated by McCarthy and Papadopoulos [15].

We also look at properties of the Weil-Petersson geodesic flow $\Phi^{t}$ on the quotient of the unit tangent bundle $T^{1} \mathcal{T}(S)$ of $\mathcal{T}(S)$ under the action of the mapping class group. Even though this quotient space $T^{1} \mathcal{M}(S)$ is non-compact and this flow is not everywhere defined, it admits many invariant Borel probability measures. Particular such measures are measures supported on periodic orbits. Each of these measures is ergodic.

The space of all $\Phi^{t}$-invariant Borel probability measures on $T^{1} \mathcal{M}(S)$ can be equipped with the weak*-topology. Our second result is a version of Theorem 1 for the Weil-Petersson geodesic flow.

Theorem 2. $A \Phi^{t}$-invariant Borel probability measure on $T^{1} \mathcal{M}(S)$ can be approximated in the weak*-topology by measures supported on periodic orbits.

The organization of this note is as follows. In Section 2 we review some geometric properties of Hadamard spaces. Section 3 explains some geometric properties of pseudo-Anosov mapping classes. In Section 4 we look at groups of isometries and establish the first and the second part of Theorem 1. In Section 5, we complete the proof of Theorem 1 and show Theorem 2.

\section{BASIC CAT(0)-GEOMETRY}

The purpose of this section is to collect some general geometric properties of CAT(0)-spaces which are needed for the investigation of Weil-Petersson space.

A CAT(0)-space is defined as follows. A triangle $\Delta$ in a geodesic metric space consists of three vertices connected by three (minimal) geodesic $\operatorname{arcs} a, b, c$. A comparison triangle $\bar{\Delta}$ for $\Delta$ in the euclidean plane is a triangle in $\mathbb{R}^{2}$ with the same side-lengths as $\Delta$. By the triangle inequality, such a comparison triangle exists always, and it is unique up to isometry. For a point $x \in a \subset \Delta$ the comparison point of $x$ in the comparison triangle $\bar{\Delta}$ is the point on the side $\bar{a}$ of $\bar{\Delta}$ corresponding to $a$ whose distance to the endpoints of $\bar{a}$ coincides with the distance of $x$ to the corresponding endpoints of $a$.

A geodesic metric space $(X, d)$ is called a $\operatorname{CAT}(0)$-space if for every geodesic triangle $\Delta$ in $X$ with sides $a, b, c$ and every comparison triangle $\bar{\Delta}$ in the euclidean plane with sides $\bar{a}, \bar{b}, \bar{c}$ and for all $x, y \in \Delta$ and all comparison points $\bar{x}, \bar{y} \in \bar{\Delta}$ we have

$$
d(x, y) \leq d(\bar{x}, \bar{y}) .
$$

A complete CAT(0)-space is called a Hadamard space.

In a Hadamard space $X$, the distance function is convex: If $\gamma, \zeta$ are two geodesics in $X$ parametrized on the same interval then the function $t \rightarrow d(\gamma(t), \zeta(t))$ is convex. For two geodesics $\gamma, \zeta$ issuing from the same point $\gamma(0)=\zeta(0)$, the Alexandrov angle 
between $\gamma, \zeta$ is defined. If $X$ is a Riemannian manifold of non-positive curvature, then this angle coincides with the angle between the tangents of $\gamma, \zeta$ at $\gamma(0)$ (see [4).

For a fixed point $x \in X$, the visual boundary $\partial X$ of $X$ is defined to be the space of all geodesic rays issuing from $x$ equipped with the topology of uniform convergence on bounded sets. This definition is independent of the choice of $x$. We denote the point in $\partial X$ defined by a geodesic ray $\gamma:[0, \infty) \rightarrow X$ by $\gamma(\infty)$. We also say that $\gamma$ connects $x$ to $\gamma(\infty)$. The union $X \cup \partial X$ has a natural topology which restricts to the usual topology on $X$ and such that $X$ is dense in $X \cup \partial X$. The isometry group of $X$ acts as a group of homeomorphisms on $X \cup \partial X$.

A subset $C \subset X$ is convex if for $x, y \in C$ the geodesic connecting $x$ to $y$ is contained in $C$ as well. For every complete convex set $C \subset X$ and every $x \in X$ there is a unique point $\pi_{C}(x) \in C$ of smallest distance to $x$ (Proposition II.2.4 of [4]). Now let $J \subset \mathbb{R}$ be a closed connected set and let $\gamma: J \rightarrow X$ be a geodesic arc. Then $\gamma(J) \subset X$ is complete and convex and hence there is a shortest distance projection $\pi_{\gamma(J)}: X \rightarrow \gamma(J)$. The projection $\pi_{\gamma(J)}: X \rightarrow \gamma(J)$ is distance nonincreasing.

The following definition is due to Bestvina and Fujiwara (Definition 3.1 of $[3]$ ).

Definition. A geodesic arc $\gamma: J \rightarrow X$ is $B$-contracting for some $B>0$ if for every closed metric ball $K$ in $X$ which is disjoint from $\gamma(J)$ the diameter of the projection $\pi_{\gamma(J)}(K)$ does not exceed $B$.

We call a geodesic contracting if it is $B$-contracting for some $B>0$. As an example, every geodesic in a $\mathrm{CAT}(\kappa)$-space for some $\kappa<0$ is $B$-contracting for a number $B=B(\kappa)>0$ only depending on $\kappa$.

The next lemma (Lemma 3.2 and 3.5 of [3]) shows that a triangle containing a $B$-contracting geodesic as one of its sides is uniformly thin.

Lemma 2.1. Let $\gamma:[a, b] \rightarrow X$ be a B-contracting geodesic. If $x \in X$ and if $a=\pi_{\gamma[a, b]}(x)$ then for every $t \in[a, b]$ the geodesic connecting $x$ to $\gamma(t)$ passes through the $3 B+1$-neighborhood of $\gamma(a)$.

On the other hand, thinness of triangles with a fixed geodesic $\gamma$ as one of the three sides guarantees that $\gamma$ is contracting. This is formulated in the following useful criterion to detect contracting geodesics. For its formuation, note that in a CAT(0)-space, the Alexandrov angle between two geodesics issuing from the same point is defined (see [4]).

Lemma 2.2. Let $\gamma: J \rightarrow X$ be a geodesic such that there is a number $B>0$ with the following property. Assume that for all $[a, b] \subset J$ with $|b-a| \geq B / 4$ and every geodesic quadrangle $Q$ in $X$ with one side $\gamma[a, b]$ and an angle at least $\pi / 2$ at $\gamma(a), \gamma(b)$ the geodesic arc connecting the two vertices of $Q$ which are distinct from $\gamma(a), \gamma(b)$ passes through the B/4-neighborhood of $\gamma[a, b]$. Then $\gamma(J)$ is Bcontracting.

Proof. Let $\gamma: J \rightarrow X$ be a geodesic which satisfies the assumption in the lemma. We have to show that $d\left(\pi_{\gamma(J)}(x), \pi_{\gamma(J)}(y)\right) \leq B$ for all $x \in X$ with $d(x, \gamma(J))=$ $R>0$ and every $y \in X$ with $d(x, y)<R$.

For this let $x \in X$ with $d(x, \gamma(J))=R>0$. Assume that $\pi_{\gamma(J)}(x)=\gamma(a)$. Let $y \in X$ and write $\pi_{\gamma(J)}(y)=\gamma(c)$ where $c \geq a$ without loss of generality (otherwise reverse the orientation of $\gamma$ ). The angle at $\gamma(c)$ between the geodesic connecting 
$\gamma(c)$ to $y$ and the subarc of $\gamma$ (with reversed orientation) connecting $\gamma(c)$ to $\gamma(a)$ is not smaller than $\pi / 2$. Since the angle sum of a triangle in a CAT(0)-space does not exceed $\pi$, this implies that if $c>a+B / 4$ then the angle at $\gamma(a+B / 4)$ of the quadrangle with vertices $x, \gamma(a), \gamma(a+B / 4), y$ is not smaller than $\pi / 2$. Thus by the assumption in the lemma, the geodesic connecting $x$ to $y$ passes through a point $z$ in the $B / 4$-neighborhood of $\gamma[a, a+B / 4]$. Then $\pi_{\gamma(J)}(z) \in \gamma[a-B / 4, a+B / 2]$, moreover also $d(x, z) \geq R-B / 4$.

Now assume that $c \geq a+B$. Then we have $d\left(\pi_{\gamma(J)}(z), \pi_{\gamma(J)}(y)\right) \geq B / 2$. Since the projection $\pi_{\gamma(J)}$ is distance non-increasing we conclude that

$$
d(x, y)=d(x, z)+d(z, y) \geq R-B / 4+B / 2>R .
$$

In other words, $\gamma(J)$ is $B$-contracting.

\section{CAT(0)-Geometry of Weil-Petersson space}

Let $S$ be an oriented surface of genus $g \geq 0$ with $m \geq 0$ punctures and $3 g-3+m \geq$ 2. The metric completion $\overline{\mathcal{T}(S)}$ of the Teichmüller space $\mathcal{T}(S)$ of $S$ with respect to the Weil-Petersson metric $d_{W P}$ is a Hadamard space. The completion locus $\overline{\mathcal{T}(S)}-\mathcal{T}(S)$ of $\mathcal{T}(S)$ can be described as follows 12 .

A surface with nodes is defined by a degenerate hyperbolic metric on $S$ where at least one essential simple closed curve on $S$ (i.e. a curve which is homotopically nontrivial and not freely homotopic into a puncture) has been pinched to a pair of punctures. For the free homotopy class of an essential simple closed curve $c$ on $S$, the degenerate surfaces with a single node at $c$ define a stratum $\mathcal{T}(S)_{c}$ in the completion locus $\overline{\mathcal{T}(S)}-\mathcal{T}(S)$ of Teichmüller space. This stratum equipped with the induced metric is isometric to the Teichmüller space equipped with the Weil-Petersson metric of the (possibly disconnected) surface obtained from $S-c$ by replacing each of the two ends corresponding to $c$ by a cusp. If $S_{c}$ is disconnected then a point in the Teichmüller space of $S-c$ is given by a pair of points, one for each of the two components of $S-c$. The stratum $\mathcal{T}(S)_{c}$ is a convex subset of $\overline{\mathcal{T}(S)}$. The completion locus $\overline{\mathcal{T}(S)}-\mathcal{T}(S)$ then is the union of the completions of the strata $\mathcal{T}(S)_{c}$ where $c$ runs through all free homotopy classes of simple closed curves and with the obvious identifications.

The extended mapping class group $\operatorname{Mod}(S)$ of all isotopy classes of diffeomorphisms of $S$ acts on $\left(\mathcal{T}(S), d_{W P}\right)$ properly discontinuously as a group of isometries. This action extends to an action on the completion $\overline{\mathcal{T}(S)}$ preserving the completion locus $\overline{\mathcal{T}(S)}-\mathcal{T}(S)$.

Masur and Wolf [13] used the action of the extended mapping class group on the completion locus of Teichmüller space to show.

Proposition 3.1. The isometry group of $\left(\mathcal{T}(S), d_{W P}\right)$ coincides with the extended mapping class group.

For $\epsilon>0$ let $\mathcal{T}(S)_{\epsilon}$ be the subset of $\mathcal{T}(S)$ of all hyperbolic metrics whose systole, i.e. the length of a shortest closed geodesic, is at least $\epsilon$. The mapping class group preserves $\mathcal{T}(S)_{\epsilon}$ and acts on it properly discontinuously and cocompactly. In particular, the Weil-Petersson distance between $\mathcal{T}(S)_{\epsilon}$ and the completion locus $\overline{\mathcal{T}(S)}-\mathcal{T}(S)$ of Teichmüller space is positive. Moreover, the sectional curvature of the restriction of the Weil-Petersson metric to $\mathcal{T}(S)_{\epsilon}$ is bounded from above by a negative constant. 
This fact together with Lemma 2.2 is used to show that Weil-Petersson geodesic segments which are entirely contained in the thick part of Teichmüller space are contracting. An analogous result for the Teichmüller metric on Teichmüller space (which is however much more difficult) was established by Minsky [16. As a convention, in the sequel a Weil-Petersson geodesic is always parametrized on a closed connected subset of $\mathbb{R}$.

Lemma 3.2. For every $\epsilon>0$ there is a number $B=B(\epsilon)>0$ such that every geodesic $\gamma: J \rightarrow \mathcal{T}(S)_{\epsilon}$ is $B$-contracting.

Proof. It was shown in Lemma 3.1 of [9] (see also [3, 7] for an earlier argument along the same line) that for every $\epsilon>0$ there is a constant $B=B(\epsilon)>0$ only depending on $\epsilon$ such that every geodesic $\gamma: J \rightarrow \mathcal{T}(S)_{\epsilon}$ satisfies the hypothesis in Lemma 2.2 for $B$.

For an isometry $g$ of $\mathcal{T}(S)$ define the displacement function $d_{g}$ of $g$ to be the function $x \rightarrow d_{g}(x)=d(x, g x)$.

Definition. An isometry $g$ of $\overline{\mathcal{T}(S)}$ is called semisimple if $d_{g}$ achieves its minimum in $\overline{\mathcal{T}(S)}$. If $g$ is semisimple and $\min d_{g}=0$ then $g$ is called elliptic. A semisimple isometry $g$ with $\min d_{g}>0$ is called axial.

By the above definition, an isometry is elliptic if and only if it fixes at least one point in $\overline{\mathcal{T}(S)}$. By Proposition 3.3 of [1, an isometry $g$ of $\overline{\mathcal{T}(S)}$ is axial if and only if there is a geodesic $\gamma: \mathbb{R} \rightarrow \overline{\mathcal{T}(S)}$ such that $g \gamma(t)=\gamma(t+\tau)$ for every $t \in \mathbb{R}$ where $\tau=\min d_{g}>0$. Such a geodesic is called an oriented axis for $g$. Note that the geodesic $t \rightarrow \gamma(-t)$ is an oriented axis for $g^{-1}$. The endpoint $\gamma(\infty)$ of $\gamma$ is a fixed point for the action of $g$ on $\partial \overline{\mathcal{T}(S)}$ which is called the attracting fixed point. The closed convex set $A \subset \overline{\mathcal{T}(S)}$ of all points for which the displacement function of $g$ is minimal is isometric to $C \times \mathbb{R}$ where $C \subset A$ is closed and convex (Theorem II.2.14 of [4]). For each $x \in C$ the set $\{x\} \times \mathbb{R}$ is an axis of $g$.

By the Nielsen-Thurston classification, a mapping class $g \in \operatorname{Mod}(S)$ either is pseudo-Anosov or it is of finite order or it is reducible. An example of a reducible mapping class is a multi-twist which can be represented in the form $\phi_{1}^{k_{1}} \circ \cdots \circ \phi_{\ell}^{k_{\ell}}$ where each $\phi_{i}$ is a Dehn-twist about a simple closed curve $c_{i}$ in $S$ and where the curves $c_{i}$ are pairwise disjoint. We allow the multi-twist to be trivial. We have.

Lemma 3.3. Every isometry $\phi$ of $\overline{\mathcal{T}(S)}$ is semi-simple, and $\phi$ is elliptic if and only if there is some $k \geq 1$ such that $\phi^{k}$ is a multi-twist.

Proof. In a Hadamard space $X$, an isometry $g$ with a finite orbit on $X$ has a fixed point which is the center of the orbit. This means the following. For a fixed orbit $\left\{x_{1}, \ldots, x_{k}\right\} \subset X$ for $g$ there is a unique point $y \in X$ such that the radius of the smallest closed metric ball centered at $y$ which contains the set $\left\{x_{1}, \ldots, x_{k}\right\}$ is minimal (Proposition II.2.7 of [4]). Since this point is defined by purely metric properties, it is a fixed point for $g$.

As a consequence, an element $g \in \operatorname{Mod}(S)$ is elliptic if and only if this holds true for $g^{k}$ for every $k>0$, and every element of finite order is elliptic.

Now assume that $g \in \operatorname{Mod}(S)$ is a multi-twist about a multi-curve $c=c_{1} \cup \cdots \cup c_{\ell}$. Let $\overline{\mathcal{T}(S)_{c}}$ be the completion of the stratum in $\overline{\mathcal{T}(S)}$ of all surfaces with nodes at the curves $c_{1}, \ldots, c_{\ell}$. Then $g$ fixes each point in $\overline{\mathcal{T}(S)_{c}}$ and hence $g$ is elliptic. 
If $g$ is pseudo-Anosov then it was shown in 8 that $g$ has an axis in $\mathcal{T}(S)$ and hence it is axial. Now assume that $g$ is reducible. Then up to replacing $g$ by $g^{k}$ for some $k>0, g$ preserves a non-trivial multi-curve $c$ component-wise, and it preserves each connected component of $S-c$. Moreover, the multi-curve $c$ can be chosen in such a way that for every component $S_{0}$ of $S-c$, either $S_{0}$ is a threeholed sphere or the restriction of $g$ to $S_{0}$ is pseudo-Anosov. If $g$ is not a multi-twist then there is at least one component $S_{0}$ of $S-c$ such that the restriction of $g$ to $S_{0}$ is pseudo-Anosov. Then the restriction of $g$ to $S_{0}$ viewed as an element of the mapping class group of $S_{0}$ has an axis in $\mathcal{T}\left(S_{0}\right)$. The Weil-Petersson metric on the stratum $\mathcal{T}(S)_{c}$ induced from the Weil-Petersson metric on $S$ is the product of the Weil-Petersson metrics on the Teichmüller spaces of the connected components of $S-c$. This implies that the restriction of $g$ to $\mathcal{T}(S)_{c}$ has an axis. Now the completion $\overline{\mathcal{T}(S)_{c}} \subset \overline{\mathcal{T}(S)}$ of $\mathcal{T}(S)_{c}$ is a closed convex subset of $\overline{\mathcal{T}(S)}$. The shortest distance projection $\overline{\mathcal{T}(S)} \rightarrow \overline{\mathcal{T}(S)_{c}}$ is distance non-increasing and equivariant with respect to the action of $g$. Therefore the infimum of the displacement function $d_{g}$ of $g$ equals the infimum of $d_{g}$ on $\overline{\mathcal{T}(S)_{c}}$. Thus this infimum is a minimum and once again, $g$ is axial.

Remark: 1) The flat strip theorem (Theorem II.2.14 of 44) states that two geodesic lines in a Hadamard space $X$ whose endpoints in the boundary $\partial X$ coincide bound a flat strip. Since the sectional curvature of the Weil-Petersson metric is negative, the proof of Lemma 3.3 shows that an axial isometry of $\overline{\mathcal{T}(S)}$ which admits an axis intersecting $\mathcal{T}(S)$ is pseudo-Anosov.

2) The celebrated solution of the Nielsen realization problem states that each finite subgroup of $\operatorname{Mod}(S)$ has a fixed point in $\mathcal{T}(S)$ [11. The discussion in the proof of Lemma 3.3 immediately implies that such a group has a fixed point in $\overline{\mathcal{T}(S)}$. It is not difficult to establish that there is also a fixed point in $\mathcal{T}(S)$, however we omit this discussion here.

The following definition is due to Bestvina and Fujiwara (Definition 5.1 of [3]).

Definition. An isometry $g$ of a CAT(0)-space $X$ is called $B$-rank-one for some $B>0$ if $g$ is axial and admits a $B$-contracting axis.

We call an isometry $g$ rank-one if $g$ is $B$-rank-one for some $B>0$. Since a pseudo-Anosov element has an axis $\gamma$ in $\mathcal{T}(S)$, by invariance and cocompactness of the action of $g$ on $\gamma$, the geodesic $\gamma$ entirely remains in $\mathcal{T}(S)_{\epsilon}$ for some $\epsilon>0$. Thus the following result (Proposition 8.1 of [3] ) is an immediate consequence of Lemma 3.2 .

Proposition 3.4. A pseudo-Anosov element in $\operatorname{Mod}(S)$ is rank-one.

Example: An axial isometry $g$ of $\overline{\mathcal{T}(S)}$ which admits an axis $\gamma$ bounding a flat half-plane is not rank-one. An example of such an axial isometry of $\overline{\mathcal{T}(S)}$ can be obtained as follows. Let $c$ be a simple closed separating curve on $S$ such that none of the two components $S_{1}, S_{2}$ of $S-c$ is a three-holed sphere. Let $g \in \operatorname{Mod}\left(S_{1}\right)$ be a pseudo-Anosov mapping class. Then $g$ defines a reducible element in $\operatorname{Mod}(S)$. If $\gamma_{1}$ is the axis for the action of $g$ on $\mathcal{T}\left(S_{1}\right)$ then for each point $z \in \mathcal{T}\left(S_{2}\right)$ the curve $\left(\gamma_{1}, z\right)$ is an axis for the action of $g$ on the stratum $\mathcal{T}(S)_{c} \subset \overline{\mathcal{T}(S)}$ and hence $\left(\gamma_{1}, z\right)$ is an axis for the action of $g$ on $\overline{\mathcal{T}(S)}$. In particular, for every infinite geodesic $\zeta: \mathbb{R} \rightarrow \mathcal{T}\left(S_{2}\right)$ the set $\{(\gamma(t), \zeta(s)) \mid s, t \in \mathbb{R}\} \subset \mathcal{T}\left(S_{1}\right) \times \mathcal{T}\left(S_{2}\right) \subset \overline{\mathcal{T}(S)}$ is an 
isometrically embedded euclidean plane in $\overline{\mathcal{T}(S)}$ containing an axis for $g$. Thus $g$ is axial but not rank-one.

A homeomorphism $g$ of a topological space $K$ is said to act with north-south dynamics if there are two fixed points $a \neq b \in K$ for the action of $g$ such that for every neighborhood $U$ of $a, V$ of $b$ there is some $k>0$ such that $g^{k}(K-V) \subset U$ and $g^{-k}(K-U) \subset V$. The point $a$ is called the attracting fixed point for $g$, and $b$ is the repelling fixed point.

Teichmüller space equipped with the Teichmüller metric can be compactified by adding the Thurston boundary $\mathcal{P} \mathcal{M L}$ of projective measured geodesic laminations which is a topological sphere. This compactification however is different from the CAT(0)-boundary $\partial \overline{\mathcal{T}(S)}$ of $\overline{\mathcal{T}(S)}$. The action of the extended mapping class group on $\mathcal{T}(S)$ naturally extends to an action on $\mathcal{P} \mathcal{M L}$. An element $g \in \operatorname{Mod}(S)$ acts on $\mathcal{P} \mathcal{M L}$ with north-south-dynamics if and only if $g$ is pseudo-Anosov. Lemma 3.3.3 of [1] shows that a rank-one isometry of a proper Hadamard space $X$ acts on the boundary $\partial X$ with north-south dynamics. The proof of this fact given in 10 (proof of Lemma 4.4) does not use the assumption of properness of $X$. Thus we obtain.

Lemma 3.5. A rank-one isometry $g$ of $\overline{\mathcal{T}(S)}$ acts with north-south dynamics on $\partial \overline{\mathcal{T}(S)}$.

\section{Non-elementary Groups of isometries}

In this section we investigate the action on $\partial \overline{\mathcal{T}(S)}$ of non-elementary subgroups of $\operatorname{Mod}(S)$ which contain a pseudo-Anosov element. We begin with recalling some standard terminology used for groups of isometries on Hadamard spaces.

Let $G<\operatorname{Mod}(S)$ be any subgroup. The limit set $\Lambda$ of $G$ is the set of accumulation points in $\partial \overline{\mathcal{T}(S)}$ of one (and hence every) orbit of the action of $G$ on $\overline{\mathcal{T}(S)}$. If $g \in G$ is axial with axis $\gamma$, then $\gamma(\infty), \gamma(-\infty) \in \Lambda$. In other words, the two fixed points for the action of a pseudo-Anosov element on $\partial \overline{\mathcal{T}(S)}$ are contained in $\Lambda$.

Lemma 4.1. The limit set of $\operatorname{Mod}(S)$ is the entire boundary $\partial \overline{\mathcal{T}(S)}$ of $\overline{\mathcal{T}(S)}$.

Proof. For suficiently small $\epsilon>0$, the set $\mathcal{T}(S)_{\epsilon} \subset \mathcal{T}(S)$ of all hyperbolic metrics whose systole is at least $\epsilon$ is connected, and the mapping class group $\operatorname{Mod}(S)$ acts cocompactly on $\mathcal{T}(S)_{\epsilon}$. There is a number $R_{0}>0$ such that the Weil-Petersson distance between any point in $\overline{\mathcal{T}(S)}$ and $\mathcal{T}(S)_{\epsilon}$ is at most $R_{0}$ [17]. Thus there is a number $R_{1}>R_{0}$ such that for all $x \in \mathcal{T}(S)_{\epsilon}$ and all $y \in \overline{\mathcal{T}(S)}$ there is some $g \in \operatorname{Mod}(S)$ with $d(g x, y) \leq R_{1}$. This just means that $\partial \overline{\mathcal{T}(S)}$ is the limit set of $\operatorname{Mod}(S)$.

Lemma 4.2. Let $G<\operatorname{Mod}(S)$ be a subgroup which contains a pseudo-Anosov element $\mathrm{g}$. Then the limit set $\Lambda$ of $G$ is the closure in $\partial \overline{\mathcal{T}(S)}$ of the set of fixed points of conjugates of $g$ in $G$. If $G$ is non-elementary then $\Lambda$ does not have isolated points.

Proof. Let $G<\operatorname{Mod}(S)$ be a subgroup which contains a pseudo-Anosov element $g \in G$. Let $\Lambda$ be the limit set of $G$. We claim that $\Lambda$ is contained in the closure of the $G$-orbit of the two fixed points of $g$. For this let $\gamma$ be the axis of $g$. By Proposition 3.4 $\gamma$ is $B$-contracting for some $B>0$. Let $\xi \in \Lambda$ and let $\left(g_{i}\right) \subset G$ be a sequence such that $\left(g_{i} \gamma(0)\right)$ converges to $\xi$. There are two cases possible. 
In the first case, up to passing to a subsequence, the geodesics $g_{i} \gamma$ eventually leave every bounded set. Let $x_{0}=\gamma(0)$ and for $i \geq 1$ let $x_{i}=\pi_{g_{i} \gamma(\mathbb{R})}(\gamma(0))$. Then $d\left(x_{0}, x_{i}\right) \rightarrow \infty(i \rightarrow \infty)$. On the other hand, $g_{i} \gamma$ is $B$-contracting and hence by Lemma 2.1 a geodesic $\zeta_{i}$ connecting $x_{0}$ to $g_{i} x_{0}$ passes through the $3 B+1$ neighborhood of $x_{i}$, and the same is true for a geodesic $\eta_{i}$ connecting $x_{0}$ to $g_{i} \gamma(\infty)$. By CAT(0)-comparison, the angles at $x_{0}$ between the geodesics $\zeta_{i}, \eta_{i}$ converge to zero as $i \rightarrow \infty$. Since $g_{i} x_{0} \rightarrow \xi$, the sequence $\left(g_{i} \gamma(\infty)\right)$ converges to $\xi$ as well. But $g_{i} \gamma(\infty)$ is a fixed point of the conjugate $g_{i} g g_{i}^{-1}$ of $g$. Thus $\xi$ is contained in the closure of the fixed points of all conjugates of $g$.

In the second case there is a bounded neighborhood $K$ of $x_{0}$ in $\overline{\mathcal{T}(S)}$ such that $g_{i} \gamma \cap K \neq \emptyset$ for all $i$. For $i>0$ let $\zeta_{i}$ be the geodesic connecting $x_{0}$ to $g_{i} x_{0}$. Since $g_{i} x_{0} \rightarrow \xi$, the geodesics $\zeta_{i}$ converge as $i \rightarrow \infty$ locally uniformly to the geodesic ray connecting $x_{0}$ to $\xi$.

After passing to a subsequence and perhaps a change of orientation of $\gamma$ we may assume that for large $i$ the point $g_{i} x_{0}$ lies between a point $z_{i} \in g_{i} \gamma \cap K$ and $g_{i} \gamma(\infty)$. This means that $g_{i} x_{0}$ is contained in the geodesic connecting $z_{i}$ to $g_{i} \gamma(\infty)$. Since the distance between $z_{i}$ and $x_{0}$ is uniformly bounded, by CAT(0)-comparison the Alexandrov angle at $g_{i} x_{0}$ between the inverse of the geodesic $\zeta_{i}$ (which connects $g_{i} x_{0}$ to $x_{0}$ ) and the inverse of the geodesic $g_{i} \gamma$ (which connects $g_{i} x_{0}$ to $z_{i}$ ) tends to zero as $i \rightarrow \infty$. This implies that the angle at $g_{i} x_{0}$ of the ideal triangle in $\overline{\mathcal{T}(S)}$ with vertices $x_{0}, g_{i} x_{0}, g_{i} \gamma(\infty)$ tends to $\pi$ as $i \rightarrow \infty$.

Since in a CAT(0)-space the sum of the Alexandrov angles of a geodesic triangle (with possibly one vertex at infinity) does not exceed $\pi$, the angle at $x_{0}$ between the geodesic $\zeta_{i}$ and the geodesic $\rho_{i}$ connecting $x_{0}$ to $g_{i} \gamma(\infty)$ tends to zero as $i \rightarrow \infty$. But $g_{i} x_{0} \rightarrow \xi$ and therefore the points $g_{i} \gamma(\infty)$ converge to $\xi(i \rightarrow \infty)$ in $\partial \overline{\mathcal{T}(S)}$. Thus $\xi$ is indeed contained in the closure of the fixed points of conjugates of $g$.

Now assume that the limit set $\Lambda$ of $G$ contains at least 3 points. Let $g$ be any pseudo-Anosov element of $G$. Since by Lemma $3.5 \mathrm{~g}$ acts with north-south dynamics on $\partial \overline{\mathcal{T}(S)}$, the set $\Lambda$ contains at least one point $\xi$ which is not a fixed point of $g$. The sequence $\left(g^{k} \xi\right)$ consists of pairwise distinct points which converge as $k \rightarrow \infty$ to the attracting fixed point of $g$. Similarly, the sequence $\left(g^{-k} \xi\right)$ consists of pairwise distinct points which converge as $k \rightarrow \infty$ to the repelling fixed point of $g$. Moreover, by the above, a point $\xi \in \Lambda$ which is not a fixed point of a pseudo-Anosov element of $G$ is a limit of fixed points of pseudo-Anosov elements. This shows that $\Lambda$ does not have isolated points and completes the proof of the lemma.

We need the following simple (and well known to the experts) observation which parallels the properties of the action of $\operatorname{Mod}(S)$ on the space of projective measured geodesic laminations. This observation follows immediately from the work of Brock, Masur and Minsky [7].

Lemma 4.3. Let $g, h \in \operatorname{Mod}(S)$ be pseudo-Anosov elements. If there is a common fixed point for the action of $g, h$ on $\partial \overline{\mathcal{T}(S)}$ then the fixed point sets of $g, h$ coincide.

Proof. Let $g, h \in \operatorname{Mod}(S)$ be pseudo-Anosov elements and assume that there is a common fixed point for the action of $g, h$ on $\partial \overline{\mathcal{T}(S)}$. Since $g, h$ act with north-south dynamics on $\partial \overline{\mathcal{T}(S)}$, this implies that the axis $\gamma$ for $g$ and the axis $\eta$ for $h$ have a common endpoint, say $\gamma(\infty)=\eta(\infty)$. We may also assume that $\gamma(\infty)$ is the attracting fixed point for both $g, h$. 
By Theorem 1.5 of [7, up to a reparametrization we have $d_{W P}(\gamma(t), \eta(t)) \rightarrow 0$ $(t \rightarrow \infty)$. After another reparametrization, there is a number $r>0$ such that the semi-group $\left\{g^{k} \mid k \geq 0\right\}$ acts cocompactly on the closed $2 r$-neighborhood $N \subset \mathcal{T}(S)$ of $\gamma[0, \infty)$, and that the $r$-neighborhood of $\gamma[0, \infty)$ contains $\eta[0, \infty)$.

Let $\tau_{0}$ be the translation length of $g$ on $\gamma$. If there are no integers $k, \ell>0$ such that $g^{k}=h^{\ell}$ then there are infinitely many distinct elements of $\operatorname{Mod}(S)$ of the form $g^{-m} h^{n}$ which map $\eta(0)$ into the $r$-neighborhood of $\gamma\left[0, \tau_{0}\right]$. Namely, let $n>0$ be arbitrary. Then there is a unique number $m \in \mathbb{Z}$ such that $\pi_{\gamma(\mathbb{R})} h^{n}(\eta(0)) \in$ $\gamma\left[m \tau_{0},(m+1) \tau_{0}\right)$. Since the point $h^{n}(\eta(0))$ is contained in the $r$-neighborhood of $\gamma(\mathbb{R})$, the point $g^{-m} h^{n}(\eta(0))$ is contained in the $r$-neighborhood of $\gamma\left[0, \tau_{0}\right]$. However, this violates the fact that $\operatorname{Mod}(S)$ acts properly discontinuously on $\mathcal{T}(S)$. Thus there are number $k, \ell>0$ with $g^{k}=h^{\ell}$ and hence the fixed point sets for the action of $g, h$ on $\partial \overline{\mathcal{T}(S)}$ coincide.

The action of a group $G$ on a topological space $Y$ is called minimal if every $G$-orbit is dense.

Lemma 4.4. Let $G<\operatorname{Mod}(S)$ be a non-elementary group with limit set $\Lambda$ which contains a pseudo-Anosov element $g \in G$ with fixed points $a \neq b \in \Lambda$. Then for every non-empty open set $V \subset \Lambda$ there is some $u \in G$ with $u\{a, b\} \subset V$. Moreover, the action of $G$ on $\Lambda$ is minimal.

Proof. Let $G<\operatorname{Mod}(S)$ be a non-elementary subgroup with limit set $\Lambda$ which contains a pseudo-Anosov element $g \in G$. Let $a, b \in \Lambda$ be the attracting and repelling fixed points of $g$, respectively, and let $V \subset \Lambda$ be a non-empty open set. By Lemma 4.2, the limit set $\Lambda$ does not have isolated points and up to replacing $g$ by $g^{-1}$ (and exchanging $a$ and $b$ ) there is an element $v \in G$ which maps $a$ to $v(a) \in V-\{a, b\}$. Then $h=v g v^{-1}$ is a pseudo-Anosov element with fixed points $v(a) \in V-\{a, b\}, v(b) \in \Lambda$. By Lemma 4.3 we have $v(b) \notin\{a, b\}$. By Lemma 3.5. $h$ acts with north-south dynamics on $\partial \overline{\mathcal{T}(S)}$ and hence $h^{k}\{a, b\} \subset V$ for all sufficiently large $k$.

Every closed $G$-invariant subset $A$ of $\partial \overline{\mathcal{T}(S)}$ contains every fixed point of every pseudo-Anosov element. Namely, if $a \neq b$ are the two fixed points of a pseudoAnosov element $g \in G$ and if there is some $\xi \in A-\{a, b\}$ then also $\{a, b\} \subset A$ since $A$ is closed and $g$ acts with north-south dynamics on $\partial \overline{\mathcal{T}(S)}$. On the other hand, if $a \in A$ then by the above consideration there is some $h \in G$ with $h(a) \in \Lambda-\{a, b\}$ and once again, we conclude by invariance that $b \in A$ as well. Now the set of all fixed points of pseudo-Anosov elements of $G$ is $G$-invariant and hence the smallest non-empty closed $G$-invariant subset of $\partial \overline{\mathcal{T}(S)}$ is the closure of the set of fixed points of pseudo-Anosov elements. This set contains the limit set $\Lambda$ of $G$ by Lemma 4.2 and hence it coincides with $\Lambda$. In other words, the action of $G$ on $\Lambda$ is minimal. The lemma is proven.

Note the following immediate corollary of Lemma 4.3

Corollary 4.5. Let $G<\operatorname{Mod}(S)$ be a non-elementary subgroup which contains a pseudo-Anosov element. Then $G$ does not fix a point in $\partial \overline{\mathcal{T}(S)}$.

Example: There are non-elementary groups $G<\operatorname{Mod}(S)$ which fix a point in $\partial \overline{\mathcal{T}(S)}$. Namely, let $c$ be a simple closed separating curve on $S$ so that $S-c=S_{1} \cup S_{2}$ 
where neither $S_{1}$ nor $S_{2}$ is a three-holed sphere. Let $\gamma_{i} \in \operatorname{Mod}\left(S_{i}\right)$ be a pseudoAnosov element $(i=1,2)$. Then $\gamma_{1}, \gamma_{2}$ generate a free abelian subgroup $G$ of $\operatorname{Mod}(S)$ whose limit set is a circle which is fixed pointwise by $G$.

We are now ready to show.

Proposition 4.6. Let $G<\operatorname{Mod}(S)$ be a non-elementary subgroup with limit set $\Lambda$ which contains a pseudo-Anosov element.

(1) The pairs of fixed points of pseudo-Anosov elements of $G$ are dense in $\Lambda \times \Lambda$.

(2) For any two non-empty open subsets $W_{1}, W_{2}$ of $\Lambda \times \Lambda$ there is some $g \in G$ with $g W_{1} \cap W_{2} \neq \emptyset$.

Proof. Let $G<\operatorname{Mod}(S)$ be a non-elementary subgroup with limit set $\Lambda$. Assume that $G$ contains a pseudo-Anosov element $g$ with attracting fixed point $a \in \Lambda$ and repelling fixed point $b \in \Lambda$.

Let $U \subset \Lambda \times \Lambda$ be a non-empty open set. Our goal is to show that $U$ contains a pair of fixed points of a pseudo-Anosov element. Since $\Lambda$ does not contain isolated points, for this we may assume that there are small open sets $V_{i} \subset \partial \overline{\mathcal{T}(S)}-\{a, b\}$ with disjoint closure $\overline{V_{i}}(i=1,2)$ and such that $U=V_{1} \times V_{2} \cap \Lambda \times \Lambda$.

Choose some $u \in G$ which maps $\{a, b\}$ into $V_{1}$. Such an element exists by Lemma 4.4. Then $v=u g u^{-1}$ is a pseudo-Anosov element with fixed points $u a, u b \in V_{1}$. Similarly, there is a pseudo-Anosov element $w \in G$ with both fixed points in $V_{2}$. Via replacing $v, w$ by sufficiently high powers we may assume that $v\left(\partial \overline{\mathcal{T}(S)}-V_{1}\right) \subset$ $V_{1}, v^{-1}\left(\partial \overline{\mathcal{T}(S)}-V_{1}\right) \subset V_{1}$ and that $w\left(\partial \overline{\mathcal{T}(S)}-V_{2}\right) \subset V_{2}, w^{-1}\left(\partial \overline{\mathcal{T}(S)}-V_{2}\right) \subset V_{2}$. Then we have $w v\left(\partial \overline{\mathcal{T}(S)}-V_{1}\right) \subset V_{2}$ and $v^{-1} w^{-1}\left(\partial \overline{\mathcal{T}(S)}-V_{2}\right) \subset V_{1}$. By a result of McCarthy [14, up to possibly replacing $v$ and $w$ by even higher powers we may assume that $w v$ is pseudo-Anosov. Then $w v$ acts on $\partial \overline{\mathcal{T}(S)}$ with north-southdynamics. Since $w v\left(\overline{V_{2}}\right) \subset V_{2}$ and $v^{-1} w^{-1}\left(\overline{V_{1}}\right) \subset V_{1}$, the pair of fixed points of $w v$ is necessarily contained in $V_{1} \times V_{2}$ and hence in $U$. The first part of the proposition is proven.

To show the second part of the proposition, let $W_{1}, W_{2} \subset \Lambda \times \Lambda$ be non-empty open sets. We have to show that there is some $g \in G$ such that $g W_{1} \cap W_{2} \neq \emptyset$. For this we may assume without loss of generality that $W_{1}=U_{1} \times U_{2}, W_{2}=U_{3} \times U_{4}$ where $U_{1}, U_{2}$ and $U_{3}, U_{4}$ are non-empty open subsets of $\Lambda$ with disjoint closure. Since $\Lambda$ does not have isolated points, by possibly replacing $U_{i}$ by proper nonempty open subsets we may assume that the sets $U_{i}$ are pairwise disjoint.

By the first part of the proposition, there is a pseudo-Anosov element $u \in G$ with attracting fixed point in $U_{1}$ and repelling fixed point in $U_{4}$. Since $u$ acts on $\partial \overline{\mathcal{T}(S)}$ with north-south dynamics, there is some $k>0$ and a small open neighborhood $U_{5} \subset U_{1}$ of the attracting fixed point of $u$ such that $u^{-k}\left(U_{5} \times U_{2}\right) \subset U_{1} \times U_{4}$. The same argument produces an element $w \in G$, a number $\ell>0$ and an open subset $U_{6}$ of $U_{2}$ such that $w^{\ell}\left(u^{-k}\left(U_{5} \times U_{6}\right)\right) \subset U_{3} \times U_{4}$. This completes the proof of the proposition.

As noted in the example after Corollary 4.5, in general the second part of Proposition 4.6 does not hold true for non-elementary subgroups of $\operatorname{Mod}(S)$ which do not contain a pseudo-Anosov element. 


\section{The Weil-Petersson GeOdesic FloW}

In this section we discuss some implications of the results in the previous section to the dynamics of the Weil-Petersson geodesic flow on moduli space.

Let $T^{1} \mathcal{T}(S)$ be the unit tangent bundle of $\mathcal{T}(S)$ for the Weil-Petersson metric. The Weil-Petersson geodesic flow $\Phi^{t}$ acts on $T^{1} \mathcal{T}(S)$ by associating to a direction $v$ and a number $t>0$ the unit tangent $\Phi^{t} v$ at $t$ of the geodesic with initial velocity $v$. Note that this flow is not everywhere defined due to the existence of finite length geodesics which end in a point in $\overline{\mathcal{T}(S)}-\mathcal{T}(S)$. Define $\mathcal{G} \subset T^{1} \mathcal{T}(S)$ to be the space of all directions of biinfinite geodesics, i.e. such that the flow line of $\Phi^{t}$ through a point $v \in \mathcal{G}$ is defined for all times. Note that the set $\mathcal{G}$ is invariant under the action of the extended mapping class group.

The following result is due to Wolpert [17, 18.

Lemma 5.1. $\mathcal{G}$ is a dense $G_{\delta}$-subset of $T^{1} \mathcal{T}(S)$ of full Lebesgue measure.

Proof. A direction at a point $x \in \mathcal{T}(S)$ either defines a geodesic ray (i.e. a geodesic defined on the half-line $[0, \infty)$ ) or a geodesic which ends at a point in $\overline{\mathcal{T}(S)}-\mathcal{T}(S)$. The set $\overline{\mathcal{T}(S)}-\mathcal{T}(S)$ is a countable union of closed convex strata, each of real codimension two. Since by the Cat(0)-property, any two points $x, y \in \overline{\mathcal{T}(S)}$ can be connected by a unique geodesic depending continuously on $x, y$, the set of directions of geodesics issuing from a point in $\mathcal{T}(S)$ and which terminate in the closure of a fixed stratum is a closed subset of $T^{1} \mathcal{T}(S)$ of real codimension one. Thus $\mathcal{G}$ is the complement in $T^{1} \mathcal{T}(S)$ of a countable union of closed subsets of codimension one, i.e. it is a dense $G_{\delta}$-set (we refer to [17, 18] for details).

Wolpert [17, 18] also observed that for every $x \in \mathcal{T}(S)$ the set of directions of geodesic rays issuing from $x$ has full Lebesgue measure in the unit sphere at $x$. Then $\mathcal{G}$ has full Lebesgue measure.

To each $v \in \mathcal{G}$ we can associate the ordered pair $\pi(v) \in \partial \overline{\mathcal{T}(S)} \times \partial \overline{\mathcal{T}(S)}$ of endpoints of the biinfinite geodesic $\gamma$ with initial velocity $v$ (here ordered means that $\pi(v)=(\gamma(\infty), \gamma(-\infty)))$. The map $\pi$ clearly is invariant under the action of the geodesic flow on $T^{1} \mathcal{T}(S)$ and hence it factors through a map of the quotient space $\mathcal{G} / \Phi^{t}$. Since the Weil-Petersson metric is negatively curved, by the flat strip theorem (Theorem II.2.13 of [4) the induced map $\mathcal{G} / \Phi^{t} \rightarrow \pi(\mathcal{G}) \subset \partial \overline{\mathcal{T}(S)} \times \partial \overline{\mathcal{T}(S)}$ is injective. This means that the set $\pi(\mathcal{G})$ can be equipped with two natural topologies: the topology as a quotient of $\mathcal{G}$, and the induced topology as a subset of $\partial \overline{\mathcal{T}(S)} \times$ $\partial \overline{\mathcal{T}(S)}$. We next observe that these two topologies coincide.

Lemma 5.2. The map $\pi$ factors through a $\operatorname{Mod}(S)$-equivariant homeomorphism of $\mathcal{G} / \Phi^{t}$ equipped with the quotient topology onto $\pi(\mathcal{G})$ equipped with the topology as a subset of $\partial \overline{\mathcal{T}(S)} \times \partial \overline{\mathcal{T}(S)}$.

Proof. By the definition of the topology of $\partial \overline{\mathcal{T}(S)}$, the map $\pi$ is continuous. Moreover, it is clearly equivariant under the action of $\operatorname{Mod}(S)$.

We have to show that $\pi$ is open for the topology of $\mathcal{G}$ as a subset of $T^{1} \mathcal{T}(S)$ and for the topology of $\pi \mathcal{G}$ as a subset of $\partial \overline{\mathcal{T}(S)} \times \partial \overline{\mathcal{T}(S)}$. For this let $v \in \mathcal{G}$ and let $U$ be a neighborhood of $v$ in $\mathcal{G}$. We have to find a neighborhood $V$ of $\pi(v)$ in $\partial \overline{\mathcal{T}(S)} \times \partial \overline{\mathcal{T}(S)}$ such that the unit tangent line of every biinfinite geodesic $\gamma$ whose pair of endpoints is contained in $V$ passes through $U$. 
For this let $x \in \mathcal{T}(S)$ be the footpoint of $v$. Since a geodesic depends smoothly on its initial velocity, if no such neighborhood $V$ of $\pi(v)$ exists then there is a sequence of points $\left(a_{i}, b_{i}\right) \subset \partial \overline{\mathcal{T}(S)} \times \partial \overline{\mathcal{T}(S)}$ which can be connected by a geodesic line $\gamma_{i}$ in $\overline{\mathcal{T}(S)}$ and there is a number $\epsilon>0$ such that $d_{W P}\left(x, \gamma_{i}(\mathbb{R})\right) \geq \epsilon$ for all $i$.

Let $\Delta_{i}$ be the ideal triangle in $\mathcal{T}(S)$ with vertices $x, a_{i}, b_{i}$. Since $a_{i} \rightarrow \gamma(\infty), b_{i} \rightarrow$ $\gamma(-\infty)$, the angle at $x$ of the triangle $\Delta_{i}$ converges to $\pi$ as $i \rightarrow \infty$. Connect each point on the geodesic ray from $x$ to $b_{i}$ to the point $a_{i} \in \partial \overline{\mathcal{T}(S)}$ by a geodesic ray. This defines a ruled surface in $\mathcal{T}(S)$ with smooth interior which we denote again by $\Delta_{i}$. The intrinsic Gauß curvature of this surface at a point $y$ is bounded from above by an upper bound for the curvature of the Weil-Petersson metric at $y$. Since the Weil-Petersson metric is negatively curved, there is a number $r<\epsilon$ such that the Gauß curvature of the intersection of $\Delta_{i}$ with the ball of radius $r$ about $x$ is bounded from above by $-r$. (Such a argument has been used in the literature many times. We refer to [7] for a more detailed explanation and for additional references).

Let $\zeta_{i}:[0, \infty) \rightarrow \mathcal{T}(S)$ be the side of $\Delta_{i}$ connecting $x$ to $a_{i}$. The intrinsic angle at $x$ of the triangle $\Delta_{i}$ coincides with the Weil-Petersson angle at $x$. By assumption, the distance between $x$ and $\gamma_{i}$ is at least $\epsilon>r$. If $i>0$ is sufficiently large that the angle of $\Delta_{i}$ at $x$ exceeds $\pi / 2$ then by convexity of the distance function, a geodesic in $\Delta_{i}$ for the intrinsic metric which issues from a point in $\zeta_{i}[0, r / 2]$ and is perpendicular to $\zeta_{i}$ does not intersect the side of $\Delta_{i}$ connecting $x$ to $b_{i}$. Therefore the maximal length of such a geodesic is not smaller than $r / 2$. The union of these geodesic segments is an embedded strip in $\Delta_{i}$ which is contained in the ball of radius $r$ about $x$ in $\mathcal{T}(S)$. Hence the Gauß curvature of $\Delta_{i}$ at each point in the strip is at most $-r$. Moreover, comparison with the euclidean plane shows that the area of the strip is at least $r^{2} / 4$. Since the Gauß curvature of $\Delta_{i}$ is negative, this implies that the integral of the Gauß curvature over $\Delta_{i}$ does not exceed $-r^{3} / 4$.

On the other hand, the angle of $\Delta_{i}$ at $x$ tends to $\pi$ as $i \rightarrow \infty$. Since the Gauß curvature of $\Delta_{i}$ is negative, the Gauß-Bonnet theorem shows that the integral of the Gauß curvature of $\Delta_{i}$ tends to zero as $i \rightarrow \infty$ which is a contradiction to the estimate in the previous paragraph. As a consequence, the image of the open set $U$ under the projection $\pi$ contains indeed an open subset of $\pi(\mathcal{G})$ equipped with the topology as a subspace of $\partial \overline{\mathcal{T}(S)} \times \partial \overline{\mathcal{T}(S)}$ which shows the lemma.

Let $T^{1} \mathcal{M}(S)$ be the quotient of the unit tangent bundle $T^{1} \mathcal{T}(S)$ of $\mathcal{T}(S)$ under the action of the (extended) mapping class group. By equivariance, the WeilPetersson geodesic flow projects to a flow on $T^{1} \mathcal{M}(S)$.

Every pseudo-Anosov element $g \in \operatorname{Mod}(S)$ defines a periodic orbit for the WeilPetersson flow. This periodic orbit is the projection of the unit tangent line of an axis of $g$. These are the only periodic orbits. Namely, if $\left\{\Phi^{t} v \mid t\right\}$ is a periodic orbit in $T^{1} \mathcal{M}(S)$ then there is a biinfinite Weil-Petersson geodesic $\gamma$ in $\mathcal{T}(S)$ whose unit tangent line projects to the orbit. This geodesic is invariant under an element $g \in \operatorname{Mod}(S)$. Then $g$ is axial, with axis $\gamma \subset \mathcal{T}(S)$, and hence $g$ is pseudo-Anosov.

Proposition 4.6, applied to the full mapping class group, shows together with Lemma 5.2 immediately the following result of Brock, Masur and Minsky [7.

Proposition 5.3. Periodic orbits are dense in $T^{1} \mathcal{M}(S)$.

Proof. Since the subset $\mathcal{G}$ of $T^{1} \mathcal{T}(S)$ is dense and $\operatorname{Mod}(S)$-invariant, it suffices to show that the unit tangents of all axes of all pseudo-Anosov elements are dense in $\mathcal{G}$. Now by Lemma 4.1 and Proposition 4.6 the set of pairs of endpoints of all axes 
of pseudo-Anosov elements in $\operatorname{Mod}(S)$ is dense in $\partial \overline{\mathcal{T}(S)} \times \partial \overline{\mathcal{T}(S)}$ and hence in $\pi \mathcal{G}$ and therefore the proposition follows from Lemma 5.2 .

We now use local compactness of Teichmüller space to complete the proof of Theorem 1 from the introduction.

Proposition 5.4. Let $G<\operatorname{Mod}(S)$ be a non-elementary subgroup with limit set $\Lambda$ which contains a pseudo-Anosov element. Then there is a dense orbit for the action of $G$ on $\Lambda \times \Lambda$.

Proof. Let $G<\operatorname{Mod}(S)$ be a non-elementary subgroup which contains a pseudoAnosov element. Let as before $\mathcal{G} \subset T^{1} \mathcal{T}(S)$ be the space of all directions of biinfinite Weil-Petersson geodesics in $\mathcal{T}(S)$ and let $\mathcal{G}_{0} \subset \mathcal{G}$ be the space of all directions of geodesics with both endpoints in $\Lambda$. Then $\mathcal{G}_{0}$ is a closed $G$-invariant subset of the (non-locally compact) space $\mathcal{G}$. By Lemma 5.2, the restriction to $\mathcal{G}_{0}$ of the map $\pi$ factors through a homeomorphism $\mathcal{G}_{0} / \Phi^{t} \rightarrow \pi\left(\mathcal{G}_{0}\right) \subset \Lambda \times \Lambda$. Since a pair of fixed points of a pseudo-Anosov element $g \in G$ is contained in $\pi(\mathcal{G})$, by Proposition 4.6 the set $\pi \mathcal{G}_{0}$ is dense in $\Lambda \times \Lambda$.

Let $P: T^{1} \mathcal{T}(S) \rightarrow T^{1} \mathcal{T}(S) / G=N$ be the canonical projection. We claim that for all nonempty open sets $U, V \subset N$ with $U \cap P \mathcal{G}_{0} \neq \emptyset$ and $V \cap P \mathcal{G}_{0} \neq \emptyset$ and every $t>0$ there is some $u \in U \cap P \mathcal{G}_{0}$ and some $T>t$ such that $\Phi^{T} u \in V$. For this let $\tilde{U}, \tilde{V}$ be the preimages of $U, V$ in $T^{1} \mathcal{T}(S)$. Then $\tilde{U}, \tilde{V}$ are open $G$-invariant subsets of $T^{1} \mathcal{T}(S)$. By Lemma 5.2 the projection $\pi: \mathcal{G} \rightarrow \pi \mathcal{G} \subset \partial \overline{\mathcal{T}(S)} \times \partial \overline{\mathcal{T}(S)}$ is open, there are open subsets $W_{1}, W_{2}$ of $\partial \overline{\mathcal{T}(S)} \times \partial \overline{\mathcal{T}(S)}$ with $W_{1} \cap \pi \mathcal{G} \subset \pi(\tilde{U})$, $W_{2} \cap \pi \mathcal{G} \subset \pi(\tilde{V})$ and such that the intersections of $W_{1}, W_{2}$ with $\Lambda \times \Lambda$ are non-empty.

Since $W_{i} \cap \Lambda \times \Lambda \neq \emptyset$, by the second part of Proposition 4.6 there is some $h \in G$ such that $W=W_{1} \cap h^{-1} W_{2} \neq \emptyset$ and that $W \cap \Lambda \times \Lambda \neq \emptyset$. Let $(a, b) \in W \cap \pi \mathcal{G}_{0}$ be the pair of endpoints of an axis of a pseudo-Anosov element $g \in G$. Such an element exists by the first part of Proposition 4.6. Then the axis of the conjugate $h g h^{-1}$ of $g$ in $G$ has a pair of endpoints $(h a, h b)$ in $W_{2} \cap \pi \mathcal{G}_{0}$. Since the unit tangent lines of axes of pseudo-Anosov elements which are conjugate in $G$ project to the same periodic orbit in $N$ for the Weil-Petersson flow, this implies that the projection of the unit tangent line of the axis of $g$ passes through both $U$ and $V$. In particular, for $t>0$ and for a point $x \in U \cap P \mathcal{G}_{0}$ contained in this periodic orbit, there is some $T>t$ such that $\Phi^{T} x \in V$. This shows our claim.

We use this observation to complete the proof of the corollary. Namely, the closure $\overline{P \mathcal{G}_{0}}$ of $P \mathcal{G}_{0}$ in $N$ is locally compact and separable since this is the case for $N$. Moreover, $\overline{P \mathcal{G}_{0}}$ is invariant under the Weil-Petersson flow $\Phi^{t}$. Hence we can choose a countable basis $U_{i}$ of open sets for $\overline{P \mathcal{G}_{0}}$. Let $V_{1}=U_{1}$ and for each $i \geq 2$ define inductively a nonempty open set $V_{i}$ in $\overline{P \mathcal{G}_{0}}$ with $\overline{V_{i}} \subset V_{i-1} \subset U_{1}$ and a number $t_{i}>t_{i-1}$ such that $\Phi^{t_{i}} \overline{V_{i}} \subset U_{i}$. This is possible by the above consideration and by continuity of the Weil-Petersson flow. Then $\cap_{i} \overline{V_{i}} \neq \emptyset$, and the forward $\Phi^{t}$-orbit of any point in $\cap_{i} \overline{V_{i}}$ is infinite and dense in $\overline{P \mathcal{G}_{0}}$. With the same argument we can also guarantee that the backward $\Phi^{t}$-orbit of a point $v \in \cap_{i} \overline{V_{i}}$ is infinite and dense in $\overline{P \mathcal{G}_{0}}$. But this just means that for a lift $\tilde{v}$ of $v$ the $G$-orbit of $\pi(v) \in \Lambda \times \Lambda$ is dense. This completes the proof of the corollary.

A flow $\Phi^{t}$ is called topologically transitive if it admits a dense orbit. As an immediate consequence of Lemma 5.2 and Proposition 5.4 we obtain the following result of Brock, Masur and Minsky [7]. 
Corollary 5.5. The Weil-Petersson geodesic flow on $T^{1} \mathcal{M}(S)$ is topologically transitive.

Remark: For two points $\xi \neq \eta \in \partial \overline{\mathcal{T}(S)}$ it is in general difficult to decide whether $(\xi, \eta) \in \pi \mathcal{G}$, i.e. whether there is a geodesic line connecting $\xi$ to $\eta$. However, Brock, Masur and Minsky [7] showed the following. Let $\gamma:[0, \infty) \rightarrow \mathcal{T}(S)$ be a geodesic ray such that there is a number $\epsilon>0$ and a sequence $t_{i} \rightarrow \infty$ with $\gamma\left(t_{i}\right) \in \mathcal{T}(S)_{\epsilon}$. Then $\gamma(\infty)$ can be connected to every $\xi \in \partial \overline{\mathcal{T}(S)}-\{\gamma(\infty)\}$ by a geodesic.

Even though the space $T^{1} \mathcal{M}(S)$ is non-compact and the Weil-Petersson geodesic flow $\Phi^{t}$ on $T^{1} \mathcal{M}(S)$ is not everywhere defined, it admits uncountably many invariant Borel-probability measures. Indeed, it was shown in 9 , that there is a continuous injection from the space of invariant probability measures for the Teichmüller flow into the space of invariant probability measures for the Weil-Petersson geodesic flow. Specific such measures are measures which are supported on periodic orbits. Each such measure is ergodic.

The space of $\Phi^{t}$-invariant Borel probability measures for the Weil-Petersson flow can naturally be equipped with the weak*-topology. With respect to this topology, it is a closed convex set in the topological vector space of all finite signed Borel measures on $\mathcal{T}^{1} \mathcal{M}(S)$. The extreme points of this convex set are just the ergodic measures. Thus Theorem 2 from the introduction is an immediate consequence of the following

Proposition 5.6. Any $\Phi^{t}$-invariant ergodic Borel probability measure can be approximated in the weak*-topology by measures supported on closed orbits.

Proof. Let $\nu$ be a Borel probability measure on $T^{1} \mathcal{M}(S)$ which is invariant and ergodic for the Weil-Petersson geodesic flow $\Phi^{t}$. We have to find a sequence of periodic orbits for $\Phi^{t}$ so that the normalized Lebesgue measures $\nu_{i}$ supported on these orbits converge weakly as $i \rightarrow \infty$ to $\nu$. This means that for every continuous function $f: T^{1} \mathcal{M}(S) \rightarrow \mathbb{R}$ with compact support we have

$$
\int f d \nu_{i} \rightarrow \int f d \nu
$$

For this let $v \in T^{1} \mathcal{M}$ be a (typical) density point for the measure $\nu$. By the Birkhoff ergodic theorem, we have

$$
\lim _{t \rightarrow \infty} \frac{1}{t} \int_{0}^{t} f\left(\Phi^{s} v\right) d s=\int f d \nu
$$

for every continuous function $f$ on $T^{1} \mathcal{M}(S)$ with compact support. Thus it suffices to find a sequence of numbers $t_{i} \rightarrow \infty$ and a sequence of periodic orbits for $\Phi^{t}$ which are the supports of normalized $\Phi^{t}$-invariant measures $\nu_{i}$ such that

$$
\left|\frac{1}{t_{i}} \int_{0}^{t_{i}} f\left(\Phi^{s} v\right) d s-\int f d \nu_{i}\right| \rightarrow 0(i \rightarrow \infty)
$$

for every continuous function $f: T^{1} \mathcal{M}(S) \rightarrow \mathbb{R}$ with compact support.

The Weil-Petersson metric induces a Riemannian metric and hence a distance function $d_{S}$ on (the orbifold) $T^{1} \mathcal{M}(S)$ (the so-called Sasaki metric). Since a continuous function $f: T^{1} \mathcal{M}(S) \rightarrow \mathbb{R}$ with compact support is bounded and uniformly 
continuous, for every $\epsilon>0$ there is a number $\delta>0$ depending on $f$ such that

$$
\left|\frac{1}{T} \int_{0}^{T} f\left(\Phi^{t} w\right) d t-\frac{1}{T} \int_{0}^{T} f\left(\Phi^{t} u\right) d t\right|<\epsilon
$$

whenever $w, u \in T^{1} \mathcal{M}(S)$ are such that $d_{S}\left(\Phi^{t} u, \Phi^{t} w\right)<\delta$ for all $t \in[\delta T,(1-\delta) T]$.

Since the sectional curvature of the Weil-Petersson metric is negative, comparison with the euclidean plane shows that the Sasaki distance in the covering space $T^{1} \mathcal{T}(S)$ can geometrically be estimated as follows. Let $P: T^{1} \mathcal{T}(S) \rightarrow \mathcal{T}(S)$ be the canonical projection. Then for every $\delta>0$ there is a number $R=R(\delta)>0$ with the following property. Let $w, u \in T^{1} \mathcal{T}(S)$ be two unit tangent vectors such that the flow-lines $\Phi^{t} w, \Phi^{t} u$ of $w, u$ are defined on the interval $[-R, R]$. If $d_{W P}\left(P \Phi^{t} u, P \Phi^{t} w\right) \leq 1 / R$ for all $t \in[-R, R]$ then $d_{S}(u, w)<\delta$.

Let $\gamma: \mathbb{R} \rightarrow \mathcal{T}(S)$ be a geodesic whose initial tangent $\gamma^{\prime}(0)$ is a preimage of $v$. By the above discussion and convexity of the distance function, it suffices to find a sequence of numbers $t_{i} \rightarrow \infty$ and a sequence $\left(g_{i}\right) \subset \operatorname{Mod}(S)$ of pseudo-Anosov elements with the following properties.

(1) There is a number $p>0$ such that the translation length of $g_{i}$ is contained in the interval $\left[t_{i}-p, t_{i}+p\right]$ for all $i$.

(2) For every number $\delta>0$ there is a number $T=T(\delta)>0$ not depending on $i$ such that the distance between the points $\gamma(T), \gamma\left(t_{i}-T\right)$ and the axis $\gamma_{i}$ of $g_{i}$ is at most $\delta$ for all sufficiently large $i$.

For the construction of such a sequence of pseudo-Anosov elements, note that by the Poincaré recurrence theorem we may assume that there is a sequence $t_{i} \rightarrow \infty$ such that $\Phi^{t_{i}} v \rightarrow v(i \rightarrow \infty)$. Let as before $\gamma: \mathbb{R} \rightarrow \mathcal{T}(S)$ be a geodesic whose initial velocity is a preimage of $v$ and write $x_{0}=\gamma(0)$. Then there is a sequence $\left(g_{i}\right) \subset \operatorname{Mod}(S)$ such that

$$
g_{i}^{-1} \gamma^{\prime}\left(t_{i}\right) \rightarrow \gamma^{\prime}(0)(i \rightarrow \infty)
$$

In particular, $d_{W P}\left(\gamma\left(t_{i}\right), g_{i} x_{0}\right) \rightarrow 0(i \rightarrow \infty)$.

Let $\zeta_{i}:\left[0, r_{i}\right] \rightarrow \mathcal{T}(S)$ be the geodesic connecting $x_{0}$ to $g_{i}\left(x_{0}\right)$. It follows from the above discussion that it suffices to show that for sufficiently large $i$ the element $g_{i} \in G$ is pseudo-Anosov and its axis has property 2) above where the geodesic arc $\gamma\left[0, t_{i}\right]$ in the statement is replaced by the geodesic arc $\zeta_{i}$.

For this recall from Lemma 3.3 that for each $i$, the element $g_{i} \in \operatorname{Mod}(S)$ either is axial or it is elliptic. If $g_{i}$ is axial then let $\gamma_{i}: \mathbb{R} \rightarrow \overline{\mathcal{T}(S)}$ be an oriented axis of $g_{i}$. Let $x_{i}=\pi_{\gamma_{i}(\mathbb{R})}\left(x_{0}\right)$. Then we have $\pi_{\gamma_{i}(\mathbb{R})}\left(g_{i} x_{0}\right)=g_{i} x_{i}=\gamma_{i}\left(\tau_{i}\right)$ where $\tau_{i}>0$ is the minimum of the displacement function of $g_{i}$. Note that $\tau_{i} \leq d_{W P}\left(x_{0}, g_{i} x_{0}\right) \leq t_{i}+1$ for all large $i$. If $g_{i}$ is not axial then let $x_{i}$ be a fixed point of $g_{i}$.

Consider the (possibly degenerate) geodesic quadrangle $Q_{i}$ in $\overline{\mathcal{T}(S)}$ with vertices $x_{0}, x_{i}, g_{i} x_{i}, g_{i} x_{0}$. The CAT(0)-angle of $Q_{i}$ at the vertices $x_{i}, g_{i} x_{i}$ is not smaller than $\pi / 2$. Since $d_{W P}\left(x_{0}, x_{i}\right)=d_{W P}\left(g_{i} x_{0}, g_{i} x_{i}\right)$, by convexity of the distance function the angles of $Q_{i}$ at $x_{0}, g_{i} x_{0}$ do not exceed $\pi / 2$. On the other hand, by equivariance under the action of $g_{i}$, the sum of the angles of $Q_{i}$ at $x_{0}$ and at $g_{i} x_{0}$ is not smaller than the angle at $x_{0}$ between the tangent of the geodesic arc $\zeta_{i}$ and the negative $-g_{i} \zeta_{i}^{\prime}\left(r_{i}\right)$ of the tangent of the geodesic arc $g_{i}^{-1} \zeta_{i}$. Now $\zeta_{i}^{\prime}(0) \rightarrow \gamma^{\prime}(0), g_{i}^{-1} \zeta_{i}^{\prime}\left(r_{i}\right) \rightarrow \gamma^{\prime}(0)$ and therefore this angle converges to $\pi$ as $i \rightarrow \infty$. Thus we may assume that the minimum of the two angles of $Q_{i}$ at $x_{0}, g_{i} x_{0}$ is bigger than $\pi / 4$ for all $i$. 


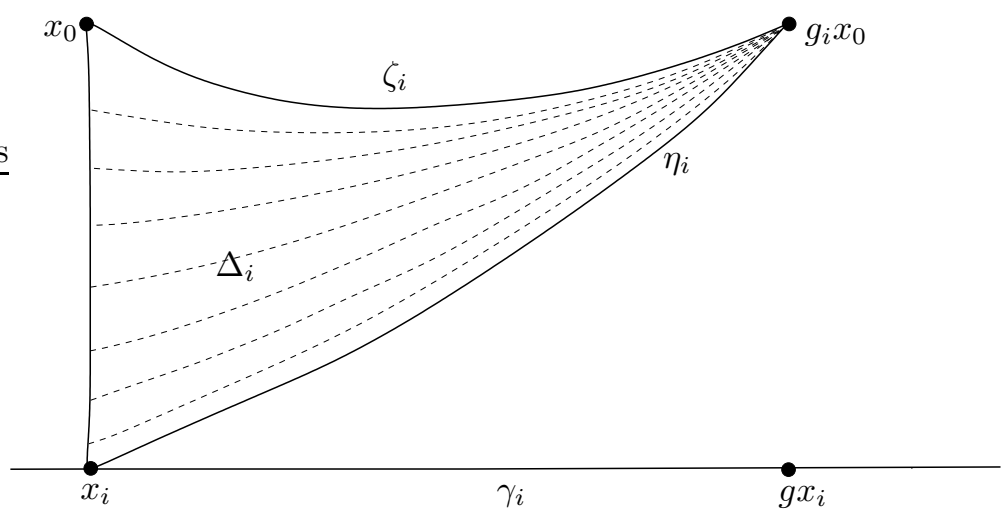

Let $\Delta_{i} \subset \overline{\mathcal{T}(S)}$ be the ruled triangle with vertices $x_{0}, x_{i}, g_{i} x_{0}$ which we obtain by connecting the vertex $g_{i} x_{0}$ to each point on the opposite side by a geodesic (see the figure). A geodesic for the Weil-Petersson metric connecting a point in $\mathcal{T}(S)$ to a point in $\overline{\mathcal{T}(S)}$ is entirely contained in $\mathcal{T}(S)$ except possibly for its endpoint [17, 18. Thus $\Delta_{i}-x_{i}$ is a smooth embedded surface in $\mathcal{T}(S)$ whose intrinsic Gauß curvature is defined. The Gauß curvature at a point $x \in \Delta_{i}$ does not exceed the maximum of the sectional curvatures of the Weil-Petersson metric at $x$ (compare the proof of Lemma 5.2 and see 7 for more and references).

Let $\epsilon>0$ be such that $x_{0} \in \mathcal{T}(S)_{2 \epsilon}$ and let $A \subset T^{1} \mathcal{M}(S)$ be the projection to $T^{1} \mathcal{M}(S)$ of the set of all unit tangent vectors in $T^{1} \mathcal{T}(S)$ with foot-point in $\mathcal{T}(S)_{\epsilon}$. Then $A$ is compact. Let $\chi$ be the characteristic function of $A$. Since $v$ is a density point for $\nu$ by assumption and since there is a neighborhood of $v$ in $T^{1} \mathcal{M}(S)$ which is entirely contained in $A$, we have $\int \chi d \nu>0$. Let $r>0$ be sufficiently small that the sectional curvature of the restriction of the Weil-Petersson metric to the $r$-neighborhood of $\mathcal{T}(S)_{\epsilon}$ is bounded from above by a negative constant $-b<0$. Such a number exists by invariance under the action of the mapping class group and cocompactness.

By CAT(0)-comparison, there is a number $s>0$ with the following property. Let $R, S>0$ and let $\zeta:[0, R] \rightarrow \overline{\mathcal{T}(S)}, \eta:[0, S] \rightarrow \overline{\mathcal{T}(S)}$ be any two geodesics issuing from $\eta(0)=\zeta(0)=x_{0}$ which enclose an angle at least $\pi / 4$. Then $\eta$ does not pass through the $r$-neighborhood of $\zeta[s, R]$. For this number $s$ and for any number $\delta<r / 3$ let $\tau=\tau(\delta)>0$ be such that $\chi\left(\Phi^{\tau} v\right)=1$ and $\int_{s}^{\tau} \chi\left(\Phi^{s} v\right) d s \geq \pi / b \delta$. Such a number exists since by the Birkhoff ergodic theorem we have

$$
\lim _{t \rightarrow \infty} \frac{1}{t} \int_{0}^{t} \chi\left(\Phi^{s} v\right) d s=\int \chi d \nu>0 .
$$

Let $i>0$ be sufficiently large that $t_{i}>\tau+2 r$, and that $d_{W P}\left(\zeta_{i}\left(r_{i}\right), \gamma\left(t_{i}\right)\right) \leq \delta / 2$. By convexity, we then have $d_{W P}\left(\zeta_{i}(t), \gamma(t)\right) \leq \delta$ for all $t \in\left[0, r_{i}\right]$. Let $\eta_{i}$ be the side of $\Delta_{i}$ connecting $x_{i}$ to $g_{i} x_{0}$. If $c$ is a geodesic arc in $\Delta_{i}$ of length at most $r$ issuing from a point in $\zeta_{i}[s, R]$ then $c$ does not intersect the side of $\Delta_{i}$ connecting $x_{i}$ to $x_{0}$ and hence either it ends on a point in $\eta_{i}$ or it can be extended. Thus if the distance between $\zeta_{i}(\tau)$ and $\eta_{i}$ is bigger than $\delta$ then by convexity the ruled triangle $\Delta_{i}$ contains an embedded strip of width $\delta<r / 3$ with the $\operatorname{arc} \zeta_{i}[s, \tau]$ as one of its sides. This strip is the union of all geodesic arcs of length $\delta$ in $\Delta_{i}$ with respect to 
the intrinsic metric which issue from a point in $\zeta_{i}[s, \tau]$ and which are perpendicular to $\zeta_{i}$. If $c$ is such a geodesic arc issuing from a point $\zeta_{i}(t)$ where $t \geq s$ is such that $\gamma_{i}^{\prime}(t) \in A$, then the Gauß curvature of $\Delta_{i}$ on each point of $c$ does not exceed $-b$. By the choice of $\tau$ and by volume comparison, the Lebesgue measure of the set of all points on such geodesic arcs is not smaller than $\pi / b$. Since the Gauss curvature of $\Delta_{i}$ is negative, this implies that the integral of the Gauss curvature of $\Delta_{i}$ over this strip is smaller than $-\pi$. However, this violates the Gauss-Bonnet theorem (compare 9] for more details for this argument, and see also the proof of Lemma 5.2).

As a consequence, the geodesic $\eta_{i}$ passes through the $\delta$-neighborhood of the point $\zeta_{i}(\tau)$. This implies that for sufficiently large $i$ the isometry $g_{i}$ is not elliptic. Namely, we have $d_{W P}\left(x_{i}, \zeta_{i}(\tau)\right) \geq d_{W P}\left(x_{0}, x_{i}\right)-\tau$ and $d_{W P}\left(x_{0}, g_{i} x_{0}\right) \geq t_{i}-\delta$ and hence if $t_{i}>2 \tau+2 \delta$ then

$$
\begin{aligned}
d_{W P}\left(x_{i}, g_{i} x_{0}\right) & \geq d_{W P}\left(x_{i}, \zeta_{i}(\tau)\right)+t_{i}-\tau-2 \delta \\
\geq d_{W P}\left(x_{i}, x_{0}\right)+t_{i}-2 \tau-2 \delta & >d_{W P}\left(x_{i}, x_{0}\right) .
\end{aligned}
$$

On the other hand, if $g_{i}$ is elliptic then we have $d_{W P}\left(x_{i}, x_{0}\right)=d_{W P}\left(g_{i} x_{i}, g_{i} x_{0}\right)=$ $d_{W P}\left(x_{i}, g_{i} x_{0}\right)$ which contradicts inequality (11). Thus $g_{i}$ is axial for all sufficiently large $i$. Let $\gamma_{i}$ be an oriented axis for $g_{i}$.

We observe next that $g_{i}$ is pseudo-Anosov for all $i$. Namely, using the above notation, let $s_{i}>0$ be such that $d_{W P}\left(\zeta_{i}(\tau), \eta_{i}\left(s_{i}\right)\right)<\delta$. If the distance between the axis $\gamma_{i}$ of $g_{i}$ and $\eta_{i}\left(s_{i}\right)$ is smaller than $\delta$ then the axis $\gamma_{i}$ of $g_{i}$ passes through the $3 \delta<r$-neighborhood of a point in $\mathcal{T}(S)_{\epsilon}$ and hence it passes through a point in $\mathcal{T}(S)$. As a consequence, $g_{i}$ is pseudo-Anosov (see the remark after Lemma 3.3).

On the other hand, if the distance between $\gamma_{i}$ and $\eta_{i}\left(s_{i}\right)$ is bigger than $\delta$ then let $T>\tau$ be such that $\int_{s}^{T} \chi\left(\Phi^{s} v\right) d s \geq 2 \pi / b \delta$ and $\chi\left(\Phi^{T} v\right)=1$. Apply the above consideration to the ruled triangle $\hat{\Delta}_{i}$ with vertices $x_{i}, g_{i} x_{i}, g_{i} x_{0}$ which we obtain by connecting $x_{i}$ to each point on the opposite side by a geodesic segment and to the subarc $\eta_{i}\left[s_{i}, s_{i}+T-\tau\right]$ of $\eta_{i}$. We conclude that $d_{W P}\left(\eta_{i}\left(s_{i}+T-\tau\right), \gamma_{i}\right)<\delta$ and once again, $\gamma_{i}$ passes through a point in $\mathcal{T}(S)$ and $g_{i}$ is pseudo-Anosov.

By the above consideration, the axis $\gamma_{i}$ of $g_{i}$ passes through the $\delta$-neighborhood of $\zeta_{i}(T)$ where $T=T(\delta)$ only depends on $\delta$. The same argument shows that this axis also passes through the $\delta$-neigbhorhood of $\zeta_{i}\left(r_{i}-\tilde{T}\right)$ where once more $\tilde{T}>0$ only depends on $\delta$ (assume without loss of generality that $-v$ is a density point for the image of $\nu$ under the flip $w \rightarrow-w$ and use the fact that two orbit segments of the same finite length are uniformly close if their initial points are close enough). In particular, the translation length of $g_{i}$ is contained in the interval $\left[t_{i}-T-\tilde{T}, t_{i}+1\right]$.

As a consequence, $\left(g_{i}\right) \subset \operatorname{Mod}(S)$ is a sequence of pseudo-Anosov elements which satisfies the conditions 1),2) above. Therefore the normalized Lebesgue measures on the projections to $T^{1} \mathcal{M}(S)$ of the unit tangent lines of the axes of the elements $g_{i}$ converge weekly to $\nu$. This completes the proof of the proposition.

\section{REFERENCES}

[1] W. Ballmann, Lectures on Spaces of Nonpositive curvature, DMV Seminar 25, Birkhäuser, Basel, Boston, Berlin 1995.

[2] W. Ballmann, M. Gromov, V. Schroeder, Manifolds of nonpositive curvature, Birkhäuser, Boston, Basel, Stuttgart 1985.

[3] M. Bestvina, K. Fujiwara, A characterization of higher rank symmetric spaces via bounded cohomology, arXiv:math/0702274, to appear in GAFA. 
[4] M. Bridson, A. Haefliger, Metric spaces of non-positive curvature, Springer, Berlin Heidelberg 1999.

[5] J. Brock, The Weil-Petersson visual sphere, Geom. Dedicata 115 (2005), 1-18.

[6] J. Brock, H. Masur, Coarse and synthetic Weil-Petersson geometry: quasi-flats, geodesics and relative hyperbolicity, Geom. Top. 12 (2008), 2453-2495.

[7] J. Brock, H. Masur, Y. Minsky, Asymptotics of Weil-Petersson geodesics I: ending laminations, recurrence, and flows, arXiv:0802.1370, to appear in GAFA.

[8] G. Daskalopoulos, R. Wentworth, Classification of Weil-Petersson isometries, Amer. J. Math. 125 (2003), 941-975.

[9] U. Hamenstädt, Invariant measures for the Weil-Petersson flow, preprint, August 2008.

[10] U. Hamenstädt, Rank-one isometries of proper CAT(0)-spaces, arXiv:0810.3794

[11] S. Kerckhoff, The Nielsen realization problem, Ann. Math. 117 (1983), 235-265.

[12] H. Masur, The extension of the Weil-Petersson metric to the boundary of Teichmüller space, Duke Math. J. 43 (1976), 623-635.

[13] H. Masur, M. Wolf, The Weil-Petersson isometry group, Geometriae Dedicata 93 (2002), $177-190$.

[14] J. McCarthy, A "Tits alternative" for subgroups of surface mapping class groups, Trans. AMS 291 (1985), 583-612.

[15] J. McCarthy, A. Papadopoulos, Dynamics on Thurston's sphere of projective measured foliations, Comm. Math. Helv. 64 (1989), 133-166.

[16] Y. Minsky, Quasi-projections in Teichmüller space, J. Reine Angew. Math. 473 (1996), $121-136$.

[17] S. Wolpert, Geometry of the Weil-Petersson completion of Teichmüller space, in "Surveys in Differential Geometry" Vol. VIII (Boston, MA, 2002), Int. Press (2003), 357-393.

[18] S. Wolpert, Convexity of the geodesic-length function: a reprise, in "Spaces of Kleinian group", London Math. Soc. Lecture Notes, Cambridge Univ. Press 2006, 233-245.

\section{MATHEMATISCHES INSTITUT DER UNIVERSITÄT BONN BERINGSTRASSE 1 \\ D-53115 BONN}

e-mail: ursula@math.uni-bonn.de 